\title{
Relativity Groups in the Presence of Matter
}

\author{
H. J. Borchers and R. N. Sen* \\ Institut für Theoretische Physik der Universität Göttingen, Göttingen, Federal Republic of Germany
}

Received October 1, 1974

\begin{abstract}
We show that the action of the boosts on an infinite system can be described continuously by bundle maps of Hilbert bundles based on the manifolds $G / G_{0}$, where $G$ is the full relativity group and $G_{0}$ its closed subgroup which can be unitarily implemented on the fibre, which is a Hilbert space. We then develop a general theory of group representations on product bundles $M \times \mathscr{H}$, where $M$ is a manifold and $\mathscr{H}$ a Hilbert space. We construct certain bundle representations of the Galilei and the Poincaré group and find that they correspond to various classes of elementary excitations. In particular, we define nonrelativistic zero-mass systems and obtain an analogue of the Faraday effect for the passage of hot electrons through matter. Our construction remains valid for the case when $G_{0}$ is the product of a lattice translation group and the time translations. We conclude that many qualitative features of the physics of condensed matter can be directly understood in terms of relativity group action on a bundle space as state space, which also suggests some avenues for further work.
\end{abstract}

\section{Contents}

Abstract . . . . . . . . . . . . . . . . . . . . . . . . . . . . . . . . . . . . 101

$\S 0$. Introduction. . . . . . . . . . . . . . . . . . . . . . . . . . . . . . . . 101

$\S 1$. Motivations . . . . . . . . . . . . . . . . . . . . . . . . . . . . . . . 102

§. Symmetries in the frame of $C^{*}$-algebras . . . . . . . . . . . . . . . . . . . . . . 103

§. Group representations on product bundles . . . . . . . . . . . . . . . . . . . . . 110

$\S 4$. Some nonrelativistic phenomena in the presence of a medium . . . . . . . . . . . . . 112

$\S 5$. The Poincaré group and homogeneous media . . . . . . . . . . . . . . . . . . . . 118

§6. Concluding remarks. . . . . . . . . . . . . . . . . . . . . . . . . . . . . . . 122

Acknowledgements . . . . . . . . . . . . . . . . . . . . . . . . . . . . . . . . 123

Appendix: Fibre Bundles . . . . . . . . . . . . . . . . . . . . . . . . . . . . . . 124

References . . . . . . . . . . . . . . . . . . . . . . . . . . . 126

\section{§ 0. Introduction}

In this article we will determine how the full Galilei or Poincaré group acts on an infinite system ${ }^{1}$. It is known that the boost operations on such systems cannot be unitarily implemented in a reasonable Hilbert space. It will turn out that the state space is a Hilbert bundle and the boost operations correspond to bundle maps which carry the fibres continuously into other fibres. A point on the base space describes a "state" of the medium or the background. We will then investigate, by an appropriate theory of group representations, the "elementary" quantummechanical objects in a "medium".

* Present address: Department of Mathematics, Technion - Israel Institute of Technology, Haifa, Israel.

1 By an infinite system we mean a system which is composed of an infinite number of massive particles. 
In Chapter 1 we discuss the considerations which motivated our work. In Chapter 2 we discuss symmetries and "broken symmetries" in the framework of $C^{*}$-algebras. We show how a continuous family of automorphisms of the algebra, which does not admit a continuous unitary representation on a Hilbert space, does admit a continuous representation on a (natural) Hilbert bundle. In Chapter 3 we develop a fairly general theory of group representations, by bundle maps, on product bundles. In Chapter 4 we determine certain bundle representations of the Galilei and the extended Galilei group. These representations appear to correspond to various collective excitations and quasi-particles in many-body systems: zero-mass excitations in superfluid helium, phonons in crystal lattices, excitations with mass in continuous and lattice systems. The significant point is that these excitations now appear as representations of the entire Galilei group, and not of just a subgroup. In Chapter 5 we shift our emphasis to localizability and discuss the "spin" 0,1 and $1 / 2$ representations of the Poincaré group on a bundle based on the boosts. The last two cases correspond precisely to "electrodynamics in a medium". We obtain an analogue of the Faraday effect for electrons. Finally, after having accumulated some material, we discuss in Chapter 6 the significance of this work and list some problems. An appendix gives a résumé of fibre bundle theory which is sufficient for our needs.

\section{§ 1. Motivations}

Our motivations for this work were threefold. First of all, we were dissatisfied with the present ideas regarding "broken symmetries". The subject came into prominence during efforts to understand broken $S U(3)$. One mechanism which was extensively studied, the "spontaneous breakdown" of internal symmetries, was shown to be equivalent to the non-implementability of the internal symmetry operation in the Hilbert space of the system. This non-implementability would not have occurred if the system had only a finite number of degrees of freedom. It was soon realised that systems with infinitely many particles should also display similar features, for example regarding the boost operations. Because of the mathematical similarities with the earlier example, this was also termed broken symmetry".

However, an internal symmetry is physically so different from a boost that we felt that the differences, and not the similarities, ought to be emphasized; the more so because one still has very little physical insight into what a "broken" internal symmetry is. The difference is, of course, that a boost operation can be "performed", in some sense; an internal symmetry operation cannot, almost by definition. We felt that it should be possible to devise a formalism in which this difference would be self-evident.

Secondly, we were interested in defining nonrelativistic zero-mass systems, which essentially meant finding a suitable state space [1]. These systems appear to exist, for example, as low-lying collective excitations in superfluid helium, the so-called phonons and rotons. Since mass cannot be defined without the boost operation, one has somehow to understand what it means to boost the background. This question is not academic because the boost properties of 
phonons and rotons may be decisive in determining the phenomena of superfluid flow. However, there exists no theoretical discussion of this suggestion, which was made by Landau [2] in 1941. The reason is that any discussion beyond Landau's original remarks would have to start from a more-or-less rigorous definition of nonrelativistic zero-mass systems and a study of their elementary properties, which so far have not been available.

Thirdly, we were interested in giving a precise formulation of the concept of a "medium" in the following context. Recent progress in the theory of condensed matter has derived largely from the picture of elementary excitations. In this picture a many-body system can often be viewed as a collection of weakly interacting excitations in the presence of a "background". The presence of the background or "medium" manifests itself in one way only; the elementary excitations cannot be "isolated" from the background, even if they are localizable. One might argue that this is no different from ordinary "atomistic" physics, because, in the same sense as above, an elementary particle cannot be "isolated" from the vacuum state. However, the vacuum state can be taken to be Poincaré invariant, and an analogous hypothesis would certainly be unacceptable for the ground state of a many-body system. Thus we still have to solve the problem of describing the kinematical properties of the ground state; a ground state is, by definition, devoid of dynamics, but not of kinematics.

It will of course not escape the reader that the above looks suspiciously like a prerequisite for studying the physics of elementary excitations ab initio and as an independent branch, with some empirical inputs such as the interaction. We were interested in finding out whether this prerequisite could be supplied.

We will terminate the discussion of motivations at this point because after a while it is no longer meaningful to distinguish between initial motivations and anticipated results. The three lines of thought raised here intersect at the same point. At this point emerges the concept of a bundle of Hilbert spaces based on a manifold. The structure of the manifold contains a complete description of the kinematics of the infinite system, or, in other words, the state of the medium. The only remark which should be added here is that product vector space bundles are physically interesting, despite their topological triviality, precisely when the base space is not a linear vector space.

\section{§ 2. Symmetries in the Frame of $\mathbf{C}^{*}$-Algebras}

\section{1. $C^{*}$-Algebras and the Symmetry Group $G$}

In the $C^{*}$-algebra frame the set of observables $\mathcal{O}$ of a physical theory is identified with the symmetric elements of a $C^{*}$-algebra $\mathscr{A}$. The set of states $\mathscr{S}$ of the system is identified with the normalized positive linear functionals on $\mathscr{A}$. Then there exists a natural map $f: \mathscr{S} \times \mathcal{O} \rightarrow \mathbb{R}$, and $f(\omega, a) \equiv(\omega, a)$, where $\omega \in \mathscr{S}, a \in \mathscr{A}$, is called the expectation value of $a$ in the state $\omega$. Since the states generate the topological dual of $\mathscr{A}, \mathscr{S}$ and $\mathcal{O}$ separate each other under $f$.

Let Aut $(\mathscr{A})$ be the group of all $*$-automorphisms of $\mathscr{A}$, and let $\alpha \in$ Aut $(\mathscr{A})$. The dual of $\alpha$ which acts on $\mathscr{S}$ is then $\alpha^{\prime}=\left(\alpha^{-1}\right)^{T}$, the superscript $T$ denoting 
transpose. Let $G$ be the symmetry group ${ }^{2}$ of the theory. For simplicity we will take $G$ to be either the Poincare or the Galilei group, excluding the space and time reflections. We will assume that $G$ is isomorphic to a subgroup of Aut $(\mathscr{A})$, and the element of Aut $(\mathscr{A})$ corresponding to $g \in G$ will be denoted by $\alpha_{g}$. We will assume that the family of maps $\alpha_{g}^{\prime}: \mathscr{S} \rightarrow \mathscr{S}$ is weakly continuous in $g$; otherwise it seems hard to understand how one can recognize a continuous symmetry group $G$ from a finite number of observations which are made with finite accuracy.

\subsection{Representations of $C^{*}$-Algebras}

A representation $\pi$ of a $C^{*}$-algebra $\mathscr{A}$ is a $*$-homomorphism of $\mathscr{A}$ into the algebra $\mathscr{B}\left(\mathscr{H}_{\pi}\right)$ of all bounded operators on the Hilbert space $\mathscr{H}_{\pi}$. We include as part of the definition the requirement that $\pi$ be non-degenerate, i.e. there exist no common null vector for the family of operators $\{\pi(a), a \in \mathscr{A}\}$. For every state $\omega$ on $\mathscr{A}$ the G.N.S. construction gives a special representation of $\mathscr{A}$ denoted by $\pi_{\omega}$.

Two representations $\pi_{1}$ and $\pi_{2}$ acting on the Hilbert spaces $\mathscr{H}_{1}$ and $\mathscr{H}_{2}$ are said to be unitary equivalent if there exists a unitary map $V: \mathscr{H}_{1} \rightarrow \mathscr{H}_{2}$ such that

$$
\pi_{1}(a)=V^{*} \pi_{2}(a) V \quad \forall a \in \mathscr{A} .
$$

Two representations $\pi_{1}$ and $\pi_{2}$ are said to be quasi-equivalent if the mapping $\phi: \pi_{1}(a) \rightarrow \pi_{2}(a)$ is an isomorphism of the algebras $\pi_{1}$ and $\pi_{2}$, and if moreover this mapping is continuous in the ultraweak topology; then the isomorphism $\phi$ extends to an isomorphism of the von Neumann algebras generated by $\pi_{1}(\mathscr{A})$ and $\pi_{2}(\mathscr{A})$. Finally, two representations $\pi_{1}$ and $\pi_{2}$ are weakly equivalent (physically equivalent in the language of Haag and Kastler [3]) if the algebras $\pi_{1}(\mathscr{A})$ and $\pi_{2}(\mathscr{A})$ are isomorphic, i.e. if the two representations $\pi_{1}$ and $\pi_{2}$ are equally faithful.

Unitary equivalence implies quasi-equivalence, which in turn implies weak equivalence. The converse implications are not generally true, i.e. these three notions of equivalence are ordered according to strength.

If one is not very familiar with the theory of $C^{*}$-algebras it may be difficult to follow the implications of the last statements. We shall remind the reader of a few definitions and results in order to make this easier.

Let $\mathscr{B}(\mathscr{H})$ be the set of bounded operators acting on the Hilbert space $\mathscr{H}$. On $\mathscr{B}(\mathscr{H})$ there exist several well-known topologies, namely the norm topology, the strong and the ultrastrong topology and the weak and the ultraweak topology. If $\pi$ is a representation of $\mathscr{A}$ acting on $\mathscr{H}$, then we can give $\pi(\mathscr{A})$ the ultraweak topology. The states which are also states of $\pi(\mathscr{A})$ and which are continuous in the ultraweak topology are called the normal states of $\pi(\mathscr{A})$. The union of these states is called by Kastler the folium of $\pi(\mathscr{A})$. The folium of $\pi(\mathscr{A})$ is the closure, in the norm topology, of the convex combinations of the vector states of $\pi(\mathscr{A})$.

The set of normal states of $\pi(\mathscr{A})$ depends on $\pi(\mathscr{A})$ and characterizes $\pi(\mathscr{A})$ up to quasi-equivalence. This set, as we shall see below, also plays a central role in characterizing those automorphisms which can be implemented by unitary operators on the representation space.

2 We will not discuss this concept, because our main aim in this chapter is to clarify the difference between the conventional notions of symmetry and broken symmetry for infinite systems. 


\subsection{The Action of $G$ on the Representations of $\mathscr{A}$}

In order to avoid unnecessary complications we shall assume that our systems are neither classical nor partly-classical and partly-quantum-mechanical, so that our $C^{*}$-algebra contains no centre. We will even go one step further and assume that the algebra $\mathscr{A}$ is simple, which means that it contains no nontrivial two-sided ideal. (This last assumption seems to be fulfilled in all concrete quantummechanical systems and is partly proved for relativistic quantum systems [4].) This means that the kernel of every representation $\pi(\mathscr{A})$ vanishes, i.e. any two representations are equally faithful or weakly equivalent ${ }^{3}$. That is, we shall be dealing with representations which are all weakly equivalent to each other.

In such a simplified situation it seems unnecessary to investigate all representations. Since the algebra $\mathscr{A}$ has no centre we shall also assume that the von Neumann algebra generated by a representation $\pi(\mathscr{A})$ has no centre, or, in other words, we shall deal only with factor representations. Summing or integrating over such representations will be done only when required by special circumstances.

We are now ready to begin discussing some situations which arise in physics.

Let $\pi(\mathscr{A})$ be a given factor representation of $\mathscr{A}$ on $\mathscr{H}_{\pi}$, and let $g \in G$. Then for $a \in \mathscr{A}$ the map

$$
a \rightarrow \pi\left(\alpha_{g} a\right) \equiv\left(\hat{\alpha}_{g} \pi\right)(a)
$$

defines another representation $\hat{\alpha}_{g} \pi$ on $\mathscr{H}_{\pi}$. Since $\pi$ and $\hat{\alpha}_{g} \pi$ are weakly equivalent, there are two possibilities regarding their quasi-equivalence:

(A) $\pi$ and $\hat{\alpha}_{g} \pi$ are quasi-equivalent for all $g \in G$.

(B) There exists a $g \in G$ such that $\pi$ and $\hat{\alpha}_{g} \pi$ are not quasi-equivalent.

Since $\pi$ and $\hat{\alpha}_{g} \pi$ are factor representations, if they are not quasi-equivalent then they are completely disjoint.

We shall call (A) and (B) the cases of $\pi$-preserved and $\pi$-broken symmetries respectively, and shall discuss them in turn. (Usually one refers to "preserved" and "broken" symmetries; we consider these terms unsatisfactory, because they seem to have the wrong connotations.)

\subsection{The Case of $\pi$-preserved Symmetries}

Recall that we have excluded time reversal and that every representation is faithful. Then, roughly speaking, in case (A) every representation of $\mathscr{A}$ is quasiequivalent to one in which the automorphisms corresponding to $G$ are unitarily implementable. More precisely:

Theorem 1 [5]. Let $G$ be a locally compact group and $\alpha$ an isomorphism of $G$ into Aut $(\mathscr{A})$ for a given $C^{*}$-algebra $\mathscr{A}$. Let $\pi(\mathscr{A})$ be a representation of $\mathscr{A}$. Then there exists a representation $\pi_{1}(\mathscr{A})$ which is quasi-equivalent to $\pi(\mathscr{A})$ and a continuous unitary representation $U(g)$ of $G$ such that

$$
\pi\left(\alpha_{g} a\right)=U(g) \pi_{1}(a) U(g)^{-1}, \quad \forall a \in A, \quad g \in G
$$

3 It is known that, according to Haag and Kastler (loc. cit.), two weakly equivalent representations contain the same physical information. However, as we shall see below, this does not imply that they are equally well adapted to display certain features of the theory. 
if and only if the following conditions are satisfied:

(i) The transpose $\alpha_{g}^{T}$ of the automorphism $\alpha_{g}$ maps the set of normal states of $\pi(\mathscr{A})$ into itself.

(ii) The action of $\alpha_{g}^{T}$ is strongly continuous on the set of normal states. That is, if $\omega$ is a normal state of $\pi(\mathscr{A})$ and $\varepsilon>0$, then there exists a neighbourhood $\mathfrak{A}$ of the identity of $G$ such that

$$
\left\|\alpha_{g}^{T} \omega-\omega\right\|<\varepsilon \text { for } g \in \mathfrak{A}(\varepsilon, \omega) .
$$

Earlier we restricted ourselves, by assumption, to factor representations and we required the action of $\alpha_{g}$ to be weakly continuous on the algebra. Now let us discuss this assumption in view of the last theorem.

Since two quasi-equivalent representations have the same set of normal states, it is always permissible to pass to a quasi-equivalent representation so that Theorem 1 applies. We therefore have only to discuss the conditions of this theorem.

(i) As $G$ is either Poincaré or Galilei, it is locally compact.

(ii) The quasi-equivalence of $\pi$ and $\hat{\alpha}_{g} \pi$ is an assumption on the choice of $\pi$ which cannot be deduced from anything else. However, such a choice is natural because we are mostly interested in representations having an invariant normal (equilibrium) state. If this is the case then this assumption is automatically fulfilled.

(iii) The continuity requirement of the theorem is somewhat stronger than necessary. If we require only the expectation values $\left(\omega, \alpha_{g} a\right)$ to be continuous, then the stronger continuity assumption of Theorem 1 is implied if the representation space of $\pi(\mathscr{A})$ is assumed separable. This conclusion rests on the following theorem:

Theorem 2 [5]. Let $\mathscr{A}$ be a $C^{*}$-algebra and $G$ a locally compact group acting as a group of $*$-automorphisms on $\mathscr{A}$. Let $\pi$ be a representation of $\mathscr{A}$ and assume that $\alpha_{g}^{\prime}$ maps the set of normal states of $\pi(\mathscr{A})$ onto itself. Then each of the following two conditions imply that the action of $\alpha_{g}^{\prime}$ is strongly continuous on the set of normal states of $\pi$ :

(i) For each normal state $\omega$ of $\pi$ and each element $x$ of the von Neumann algebra generated by $\pi(\mathscr{A})$ the function $\left(\omega, \pi\left(\alpha_{g} x\right)\right)$ is continuous on $G$.

(ii) There exists a representation $\pi_{1}(\mathscr{A})$ which is quasi-equivalent to $\pi(\mathscr{A})$ and acts on a separable Hilbert space. The functions $\left(\omega, \pi\left(\alpha_{g} x\right)\right)$ are continuous for $x \in \mathscr{A}$ and $\omega$ a normal state of $\pi(\mathscr{A})$.

Remark. Since the set of normal states is the norm-closure of the convex combinations of vector states of $\pi(\mathscr{A})$ (or $\pi_{1}(\mathscr{A})$ ), it is sufficient to require the continuity only for vector states of $\pi(\mathscr{A})$ or $\pi_{1}(\mathscr{A})$, or any other representation quasi-equivalent to $\pi(\mathscr{A})$.

This concludes the discussion of $\pi$-preserved symmetries.

\subsection{The Case of $\pi$-broken Symmetries}

If the symmetry is $\pi$-broken the action $\hat{\alpha}_{g}$ on the space of weakly equivalent factor representations is such that $\pi$ is not quasi-equivalent to $\hat{\alpha}_{g} \pi$ for at least one 
$g \in G$. Choose a representation $\pi_{0}$ and denote by $M$ the orbit generated by the action of the $\hat{\alpha}_{g}$ on $\pi_{0}$. Denote the elements of $M$ by $m$ and in particular the element $\pi_{0}$ by $m_{0}$. Furthermore let $G_{m},\left(G_{0}\right)$ be the subgroup of $G$ such that $\hat{\alpha}_{g} \pi_{m},\left(\hat{\alpha}_{g} \pi_{0}\right)$ is quasi-equivalent to $\pi_{m},\left(\pi_{0}\right)$. If $m \in M$ is different from $m_{0}$ then there exist $g \in G$ such that $\pi_{m}=\hat{\alpha}_{g} \pi_{0}$. From this follows that the groups $G_{m}$ and $G_{0}$ are conjugate to each other:

$$
G_{m}=g^{-1} G_{0} g
$$

(Since we have defined $\left(\hat{\alpha}_{g} \pi\right)(x)=\pi\left(\alpha_{g} x\right)$ we will deal with right cosets. If one prefers left cosets one should have defined $\left(\tilde{\alpha}_{g} \pi\right)(x)=\pi\left(\alpha_{g^{-1}} x\right)$.

Let now $g_{1}, g_{2} \in G$ such that $\hat{\alpha}_{g_{1}} \pi_{0} \cong \hat{\alpha}_{g_{2}} \pi_{0}$ ( $\cong$ denotes quasi-equivalence). Then $\pi_{0}\left(\alpha_{g_{1}} x\right) \cong \pi_{0}\left(\alpha_{g_{2}} x\right)$. Replacing $x$ by $\alpha_{g_{2}-1} x$ we obtain $\pi_{0}\left(\alpha_{g_{1} g_{2}{ }^{-1}} x\right) \cong \pi_{0}(x)$, so that $\hat{\alpha}_{g_{1}} \pi_{0} \cong \hat{\alpha}_{g_{2}} \pi_{0}$ if and only if $g_{1} g_{2}^{-1} \in G_{0}$. In other words, there exists a pointwise correspondence between the orbit $M$ and the space of right cosets of $G_{0}$. Since $G$ is locally compact, the latter carries a Hausdorff topology if $G_{0}$ is a closed subgroup of $G$. We shall assume this to be true.

We shall use the same letter $m$ to denote the right coset of $G_{0}$ which corresponds to the point $m$ on $M$.

Let us now see what happens if we insist on looking for unitary group representations. Since $G$ is not $\pi$-preserved, the orbit $M$ must contain more than one point. Therefore a unitary representation of $G$ can be constructed (if the situation is favourable) only in the Hilbert space

$$
\mathscr{H}=\sum_{m \in M} \oplus \mathscr{H}_{m}
$$

where $\mathscr{H}_{m}$ is the representation space of $\hat{\alpha}_{g} \pi_{0}$ with $g \in m$ (here $m$ is a right coset of $G_{0}$ ). In most cases of interest $M$ will be a continuum, so that $\mathscr{H}$ will become a huge Hilbert space. Assume now that there exists on $\mathscr{H}$ a unitary representation $U(g)$ implementing the automorphisms, i.e. $U(g) \pi(x) U(g)^{-1}=\pi\left(\alpha_{g} x\right)$, where $\pi$ is the representation $\pi=\sum_{m} \oplus \pi_{m}$. Let $g$ be a group element which does not belong to the "little group" $G_{m}$ of the representation $\pi_{m}$. Then $U(g)$ must map $\mathscr{H}_{m}$ onto $\mathscr{H}_{m g^{-1}}$, which is orthogonal to $\mathscr{H}_{m}$. This holds even if $g$ is arbitrarily close to the subgroup $G_{m}$, from which we are forced to conclude that the unitary representation $U(g)$ cannot be continuous (except on the normal subgroup $\left.\bigcap_{m \in M} G_{m}\right)$. Since mathematically we are not used to dealing with discontinuous representations, we are bound to lose information in cases in which the group action displays some continuity (e.g. if the functions $\left(\omega, \alpha_{g} x\right)$ are assumed continuous on $G$ ). We are convinced that such continuity assumptions should have implications for the group representations. However, looking for such implications requires techniques other than (unitary) representation theory, and we are forced to search for better mathematical concepts.

Such a concept is provided by fibre bundles.

Instead of using the orbit $M$ as an index set for summing Hilbert spaces, we shall view it as a base space and the representation space $\mathscr{H}_{m}$ as the fibre over 
$m \in M$. It now becomes clear that we want $M$ to be furnished with a nice topology, for two reasons. Firstly, we would like to work with Hilbert bundles and secondly we would like the action of $G$ on $M$ to be continuous. (We shall assume this to be true for the time being and justify it at the end of this section.) Now all our representations $\pi_{m}=\hat{\alpha}_{g} \pi_{0}, g \in m$ are representations on the same Hilbert space $\mathscr{H}_{0}=\mathscr{H}_{m_{0}}$, because $\hat{\alpha}_{g} \pi_{0}(x)$ has been defined by $\pi_{0}\left(\alpha_{g} x\right)$. This means that we are dealing with a product Hilbert bundle $M \times \mathscr{H}_{0}$. However, this could have been expected, because (see Appendix) all Hilbert bundles on paracompact bases are equivalent to the product.

In order to complete this scheme it is necessary to define an action of the group on the bundle space. This action must fulfil the following requirements. If $g$ belongs to a coset $m$ then $g$ must map the point $m_{0}$ onto $m$. If $g$ belongs to $G_{0}$ then it acts only on the fibre $\mathscr{H}_{0}$. We recognize this situation to be analogous to the situation of induced representations. Therefore we choose from every coset $m$ an element $\eta(m) \in m$. Then it follows that $m_{0} \eta(m)=m$. For the action of the group $G_{0}$ on $\mathscr{H}_{0}$ we shall assume that the conditions of Theorem 1 are satisfied, so that we can assume (eventually after passing to a representation quasi-equivalent to $\pi_{0}$ ) that we have a continuous representation $U(g)$ of $G_{0}$ acting on $\mathscr{H}$ and implementing the automorphisms $\alpha_{g}$ for $g \in G_{0}$. Now take a point $(m, \psi)$ in $M \times \mathscr{H}$, with $m \in M$ and $\psi \in \mathscr{H}$. Remark that $\eta\left(m g^{-1}\right) \cdot g \eta(m)^{-1}$ maps the right-coset $m_{0}$ onto $m_{0}$, so that the action of $G$ on $M \times \mathscr{H}$ is given by

$$
g:(m, \psi) \rightarrow\left(m g^{-1}, U\left(\eta\left(m g^{-1}\right) g \eta(m)^{-1}\right) \psi\right) .
$$

The unusual appearance of this formula is due to the use of right cosets. If we had used left cosets we would have obtained

$$
g:(m, \psi) \rightarrow\left(g m, U\left(\eta(g m)^{-1} g \eta(m)\right) \psi\right),
$$

which looks much more familiar.

Thus the group action on the bundle space contains an arbitrariness in the choice of the function $\eta(m)$. That this choice is free is best seen by passing to the "big" representation space $\mathscr{H}$ of (2.1). Identifying $\mathscr{H}_{m}$ with $\mathscr{H}_{0}$ requires fixing a map from $\mathscr{H}_{0}$ onto $\mathscr{H}_{m}$. Such a map is given in a natural manner by the action $\hat{\alpha}_{g} \pi_{0}$. Thus to identify $\mathscr{H}_{m}$ with $\mathscr{H}_{0}$ we have to choose an element $\eta(m)$ from each co-class $m$ for this identification. Any other choice will then result only in a unitary transformation in the big Hilbert space $\mathscr{H}$ which leaves every $\mathscr{H}_{m}$ fixed.

Since our basic idea was to display continuity it is natural to try to choose $\eta(m)$ as a continuous function on $M$ with values in $G$. If the action of the group $G$ on the base space $M$ is continuous, then we can begin constructing continuous group representations on the bundle space $M \times \mathscr{H}_{0}$. These questions are discussed in the next chapter.

Having constructed such a representation of $G$ on $M \times \mathscr{H}_{0}$ we can pass easily to the big Hilbert space $\mathscr{H}$ by making $M \times \mathscr{H}_{0}$ into a Hilbert space. Introduce the scalar product

$$
\left((m, \psi),\left(m^{\prime}, \psi^{\prime}\right)\right)=\delta_{m m^{\prime}}\left(\psi, \psi^{\prime}\right) .
$$


Then we naturally recover a unitary representation of $G$ on $\mathscr{H}$. But we also see at which point we have lost continuity, namely we have now furnished the orbit $M$ with the discrete topology!

On the other hand, if we start from a unitary representation on $\mathscr{H}$ such that $G_{m}$ maps $\mathscr{H}_{m}$ onto itself, then we can work backwards and construct an action of $G$ on the bundle space $M \times \mathscr{H}_{0}$, by identifying $\mathscr{H}_{0}$ with $U(\eta(m)) \mathscr{H}_{0}$. However, it is evident that not every unitary representation of $G$ on $\mathscr{H}$ with the above properties will lead to a continuous action of $G$ on $M \times \mathscr{H}_{0}$. Hence we see that bundle spaces give a natural framework for displaying continuity properties.

Finally, it remains to discuss the continuity of the action of $G$ on $M$. Recall that we started our discussion of $\pi$-broken symmetries from a given representation $\pi_{0}$ acting on a Hilbert space $\mathscr{H}_{0}$. By definition all representations $\hat{\alpha}_{g} \pi_{0}$ act on the same Hilbert space. This allows us to introduce on the space

$$
\left\{\hat{\alpha}_{g} \pi_{0} ; g \in G\right\} \cong G
$$

a topology $\tau_{1}$ by neighbourhoods characterized by $x_{1} \ldots x_{m} \in \mathscr{A}$ and $\psi_{1} \ldots \psi_{n}$ $\in \mathscr{H}_{0}$ defined by

$$
\begin{gathered}
U_{x_{1} \ldots x_{m}, \psi_{1} \ldots \psi_{n}}\left(\hat{\alpha}_{g} \pi_{0}\right)=\left\{g^{\prime} ;\left|\left(\psi_{i}\left[\hat{\alpha}_{g^{\prime}} \pi_{0}\left(x_{j}\right)-\hat{\alpha}_{g} \pi_{0}\left(x_{j}\right)\right] \psi_{i}\right)\right|<1\right. \\
\text { for } i=1, \ldots n, j=1, \ldots m\} .
\end{gathered}
$$

It is known that this defines a Hausdorff topology if $\pi_{0}$ is non-degenerate $([6]$; see also [7] III.3.5 and III.3.9.8); the latter was part of our assumptions.

If all functions $\left(\psi, \pi_{0}\left(\alpha_{g} x\right) \psi\right)$ for $x \in \mathscr{A}$ and $\psi \in \mathscr{H}_{0}$ are continuous on $G$ in its group topology $\tau_{0}$ then we find that the action of $G$ on $\left(G, \tau_{1}\right)$ defined by

is continuous.

$$
g \rightarrow \hat{\alpha}_{g} \hat{\alpha}_{g_{0}} \pi_{0}
$$

For further discussion let us assume that the given representation has an infinite commutant. In this case every normal state of $\pi_{0}$ is also a vector state and moreover if $\pi_{0}$ and $\hat{\alpha}_{g} \pi_{0}$ are quasi-equivalent then they are also unitarily equivalent. Therefore passing from $G$ to $M=G / G_{0}$ is nothing but dividing the space $\left(G, \tau_{1}\right)$ by the unitary equivalence relation. Denoting the final topology on $M$ again by $\tau_{1}$, then it is known (see [7] III.3.5.5) that the map

$$
\left(G, \tau_{1}\right) \rightarrow\left(M, \tau_{1}\right)
$$

is open. Therefore the action of $G$ on $\left(M, \tau_{1}\right)$ is again continuous.

The only problem which remains is the question of whether $\tau_{1}$ defines a Hausdorff topology on $M$. We cannot expect that this will follow from general principles, but in most cases of physical interest it will be true.

We end this discussion with a remark. We have constructed the base space $M$ in such a way that it coincides with $G / G_{0}$ as a set. But the topology $\tau_{1}$ constructed from the representation $\pi_{0}$ is not necessarily the same as the quotient space topology of $G / G_{0}$ constructed from the group topology $\tau_{0}$. 


\section{§ 3. Group Representations on Product Bundles ${ }^{4}$}

\subsection{Definition of Representation}

Let $G$ be a Lie group, $H$ a closed subgroup of $G$ and $B=M \times \mathscr{H}$ a product bundle, where $M=G / H$ is the homogeneous space (i.e. the space of left-cosets with the quotient topology) and $\mathscr{H}$ is a Hilbert (sometimes a normed finitedimensional vector) space. We define a bundle representation of $G$ on $B$ to be a family of (invertible) bundle maps

$$
h(g): B \rightarrow B, \quad g \in G
$$

satisfying:

a) $h(g)$ is continuous in $g$;

b) $h(e)=\mathrm{id}$, the identity map;

c) $h\left(g_{1}\right) h\left(g_{2}\right)=h\left(g_{1} g_{2}\right)$, for all $g_{1}, g_{2} \in G$.

It follows that $h(g)^{-1}=h\left(g^{-1}\right)$ is continuous in $g$.

We will employ the notations $x \in M, \phi \in \mathscr{H}$ and $B \ni b=(x, \phi)$. Let $\pi: B \rightarrow M$ be the natural projection $\pi(b)=x$. Since $h(g)$ is a bundle map, it induces a continuous base map $\bar{h}(g)$ under $\pi$ :

$$
\pi \circ h(b)=\bar{h} \circ \pi(b),
$$

and $\bar{h}$ satisfies $\bar{h}\left(g_{1}\right) \bar{h}\left(g_{2}\right)=\bar{h}\left(g_{1} g_{2}\right), \bar{h}(e) x=x \forall x \in M$. The triple $(G, M, \bar{h})$ is a $G$ space; it is in fact a $G$-orbit, with stabilizers conjugate to $H$. It follows quickly that $\bar{h}$ is the natural action of $G$ on $M$ :

$$
\bar{h}(g) x=\pi\left(g \pi^{-1}(x)\right) .
$$

We will denote $\bar{h}(g) x$ by $g x$, and $h(g) b$ by $g b$.

In order to be consistent with (3.3), the representation $h$ must be of the form

$$
h(b)=g(x, \phi)=(g x, u(g, x) \phi),
$$

with $u(g, x) \phi$ satisfying

(i) $u(e, x) \phi=\phi \quad \forall x \in M$ and $\phi \in \mathscr{H}$;

(ii) $u\left(g_{1}, g_{2} x\right) u\left(g_{2}, x\right) \phi=u\left(g_{1} g_{2}, x\right) \phi \quad \forall g_{1}, g_{2} \in G, x \in M$ and $\phi \in \mathscr{H}$.

Since we are working with a Hilbert space $\mathscr{H}$ we will require $u(g, x)$ to take its values in the group of unitaries on $\mathscr{H}$. Then (i) and (ii) above are replaced by

$$
\begin{aligned}
& u(e, x)=\mathbb{1} \quad \forall x \in M ; \\
& u\left(g_{1}, g_{2} x\right) u\left(g_{2}, x\right)=u\left(g_{1} g_{2}, x\right) \quad \forall g_{1}, g_{2} \in G \quad \text { and } \quad x \in M .
\end{aligned}
$$

The continuity condition ${ }^{5}$ on the representation $h$ requires that:

$$
u(g, x) \phi \quad \text { be continuous in } g \text { and } x \text { for all } \phi \in \mathscr{H} .
$$

Observe that if $u(g, x)$ is independent of $x$ for all $g$, then the structure one obtains is sometimes called a Hilbert G-bundle in the literature.

${ }^{4}$ A survey of the required material on bundles is given in the Appendix.

${ }^{5}$ Sometimes, by abuse of language, we shall speak of a bundle representation even when this representation is not continuous. 
The fundamental problem of the theory of bundle representations in the following: Given $G, H, G / H$ and $\bar{h}$ as above, find all solutions of the equations (3.5) on a Hilbert space $\mathscr{H}$ subject to the continuity condition (3.6). We shall not attampt to solve this problem in its generality, but rather shall display a class of solutions which seems to have immediate physical relevance. This class of solutions is obtained as follows.

\subsection{The Canonical Representations}

Let $x_{0}$ be the point in $M$ corresponding to the left-coset $H$ itself, and define a $(1,1)$ map

$$
\eta: M \rightarrow G
$$

satisfying

$$
\pi \eta(x)=x \quad \forall x \in M .
$$

Then $\eta(x) x_{0}=x$. Now, using $\eta$, define a map

$$
k: G \times M \rightarrow G
$$

by

$$
k(g, x)=\eta(g x)^{-1} g \eta(x) .
$$

Then $k(g, x)$ satisfies the Eq. (3.5), with $u$ replaced by $k$ and $\mathbb{1}$ replaced by $e$, and, as one readily verifies, $k(g, x)$ takes its values in $H$. Hence, a continuous unitary representation $U(g)$ of $H$ on $\mathscr{H}$ yields a bundle representation of $G$ by the identification

$$
u(g, x)=U(k(g, x)),
$$

provided that the condition (3.6) is satisfied. Now there are two possibilities:

a) The Bundle $[G, \pi, M, H, H]$ is a Product. Then cross-sections exist, and a continuous map $\eta: M \rightarrow G$ satisfying (3.7b) is nothing but a cross-section of the bundle $[G, \pi, M]$; the inverse of a cross-section is also continuous, and hence $k(g, x)$ as defined by (3.8) is continuous and there is nothing more to discuss.

b) The Bundle $[G, \pi, M, H, H]$ is not a Product. Since we have assumed $G$ to be Lie and $H$ to be a closed subgroup of $G, H$ has a local cross-section in $G$. It is therefore always possible to choose a map $\eta$ satisfying (3.7) whose discontinuities lie on a set of measure zero in $M$. The discontinuities of $k: G \times M \rightarrow H$ then also lie on a set of measure zero in $G \times M$. However, the bundle representation defined by (3.9) is still continuous, provided that we take for $\mathscr{H}$ the space $L_{2}(M, \mathrm{~d} \mu)$, where $\mathrm{d} \mu$ is a measure on $M$ invariant under the action $h(g)$ of $G$. Then for any vector $\phi \in L_{2}(M, \mathrm{~d} \mu)$, the vector

$$
U(k(g, x)) \phi
$$

is by definition unchanged by changing its values on a set of measure zero in $M$. Therefore the operator $U(k(g, x))$ is unchanged by changing $k$ on a set of measure zero on $M$. Hence it is possible to tolerate discontinuities in $k$, provided that they can be traced to a set of measure zero in $M$. 
Suppose that $H$ is Abelian. It may still happen that one can define a unitary representation of $H$ on $L_{2}(M, \mathrm{~d} \mu)$. Then this representation is reducible, but it yields a (continuous) bundle representation of $G$. In this case one cannot obtain a continuous bundle representation of $G$ from any irreducible unitary representation of $H$ (except the identity).

\subsection{Equivalence and Irreducibility}

A canonical bundle representation $h$ is synthesized out of two ingredients: the natural representation $\bar{h}$ of $G$ on $G / H$, which is defined in a coordinate-free manner, and a representation $U$ of $H$ on $\mathscr{H}$, which is not. Now let $V: B \rightarrow B$ be a bundle map which induces the identity map on the base space $M=G / H$. Then $V$ acts only as unitary transformations on the fibres. That is, (i) $V$ is a bundle equivalence, and (ii) $V$ transforms the representation $U$ of $H$ to $V U V^{-1}$. It is therefore natural to define the representations $h$ and $h \circ V$ to be equivalent. It follows from a standard result in the theory of induced representations that bundle representations $h$ and $h^{\prime}$ constructed from the same manifold $G / H$, same representation $U$ of $H$ but different $\eta$ and $\eta^{\prime}$ are equivalent in this sense.

Finally, it remains to discuss reducibility and irreducibility. We assume that $H$ is of type I, so that the representation $U$, if reducible, is fully reducible. The bundle $B$ then splits under the action of $G$ into a collection of disjoint invariant subspaces, each of which is a product based on the same manifold $G / H$, and each carries an irreducible representation of $H$ on its fibre. It is natural to call these bundle representation irreducible.

Conversely, given two irreducible bundle representations of $G$ based on the same manifold $G / H$, one can always form their "direct sum" in an obvious manner. This operation is the trivial analogue of the formation of Whitney sums of vector bundles with a common base space, trivial because the bundles here are products.

Lastly, we may relax the condition that $M$ is a homogeneous space, and replace it by an arbitrary $G$-space $X$. Then $X$ splits under $G$ into disjoint $G$-orbits. A glance at (3.4) and (3.5) reveals that $u(g, x)$ and therefore also $k(g, x)$ respect this splitting. Hence, if $X$ is an arbitrary $G$-space, one may define an irreducible bundle representation to be one which is transitive on the base and irreducible on the fibre.

If $M$ is a $G$-orbit with stabilizers conjugate to $H$ and $G$ is a product bundle over $G / H$, it is easily proved that the manifolds $M$ and $G / H$ are homeomorphic, and that this homeomorphism preserves the action of the group. In this case there is no topological reason to distinguish between $M$ and $G / H$. If $G$ is not a product bundle over $G / H$, then the continuity of the bundle representation based on $M$ must be discussed separately.

\section{$\S 4$. Some Nonrelativistic Phenomena in the Presence of a Medium}

In this chapter we will determine certain bundle representations of the Galilei group and its extension which appear to correspond to (nonrelativistic) elementary excitations in continuous and lattice systems. These representations are constructed on momentum space and we essentially avoid all questions concerning localizability, which will be discussed for the relativistic case in the next chapter. 
We will denote by $G_{3}$ the Galilei group and by $\tilde{G}_{3}$ its central extension. The elements $g \in G_{3}$ will be parametrized as $g=(b, \boldsymbol{a}, \boldsymbol{v}, R)$, where $b$ is a time translation, $\boldsymbol{a}$ a space translation, $\boldsymbol{v}$ a boost and $R$ a (proper) rotation. The multiplication law in $G_{3}$ is

$$
\left(b^{\prime}, \boldsymbol{a}^{\prime}, \boldsymbol{v}^{\prime}, R^{\prime}\right)(b, \boldsymbol{a}, \boldsymbol{v}, R)=\left(b^{\prime}+b, \boldsymbol{a}^{\prime}+R^{\prime} \boldsymbol{a}+\boldsymbol{v}^{\prime} b, \boldsymbol{v}^{\prime}+R^{\prime} \boldsymbol{v}, R^{\prime} R\right) .
$$

An element of $\tilde{G}_{3}$ will be denoted by $\tilde{g}=(\theta, g)=(\theta, b, \boldsymbol{a}, \boldsymbol{v}, R)$. The multiplication law in $\tilde{G}_{3}$ is

$$
\tilde{g}^{\prime} \tilde{g}=\left(\theta^{\prime}, g^{\prime}\right)(\theta, g)=\left(\theta^{\prime}+\theta+\xi\left(g^{\prime}, g\right), g^{\prime} g\right)
$$

where

$$
\xi\left(g^{\prime}, g\right)=\frac{1}{2} \boldsymbol{v}^{\prime 2} b+\boldsymbol{v}^{\prime} \cdot R^{\prime} \boldsymbol{a} .
$$

We will denote by $T$ the group of time translations, and by $S_{j}, V_{j}, R_{j}, E_{j}$ respectively the groups of space translations, boosts, rotations and Euclidean operations in $j$ dimensions $(j=2,3) . A^{*}$ will denote the covering group of $A$, which is two-sheeted for the rotation group. (The groups $S_{2,3}$ should not be confused with the spheres $S_{2,3}$.)

\subsection{Nonrelativistic Zero-Mass Systems}

These systems appear to exist as elementary excitations on a translationally invariant ground state. The most important of these is probably the phononroton branch of the excitation spectrum of superfluid helium. They correspond to bundle representations of $G$ on $G / H$ where $G=G_{3}^{*}$ and $H=H_{3}^{*} \times T$. Clearly $G_{3}^{*} / E_{3}^{*} \times T=G_{3} / E_{3} \times T \cong V_{3}$, which is paracompact and contractible. Hence $G_{3}$ is a product bundle over $V_{3}$ and cross-sections exist.

Let $w \in V_{3}$. The action of the group on the manifold is

$$
(b, \boldsymbol{a}, \boldsymbol{v}, R) \boldsymbol{w}=\boldsymbol{v}+R \boldsymbol{w}
$$

and there exists the natural cross-section

$$
\eta(w)=(0,0, w, 1) .
$$

Hence

$$
k(g, \boldsymbol{w})=(b, \boldsymbol{a},-b(\boldsymbol{v}+R \boldsymbol{w}), 0, R)
$$

where $g=(b, \boldsymbol{a}, \boldsymbol{v}, R)$ and $k$ is defined by (3.8).

We choose the following representation of the group $E_{3} \times T \ni(b, a, 0, R)$ :

$$
U(b, \boldsymbol{a}, 0, R) \phi(\boldsymbol{p})=\exp \operatorname{inr}(\omega, R) \exp i(b E-\boldsymbol{a} \cdot \boldsymbol{p}) \phi \circ R^{-1}(\boldsymbol{p}) .
$$

Here (i) $n$ is an integer or half-integer, positive, negative or zero; (ii) $r(\omega, R)$ is a Wigner rotation corresponding to $R$ and $\omega$ is the point $p$ on the sphere $S_{2}\left(p^{2}=1\right)$; (iii) $\phi \in L_{2}\left(\mathbb{R}^{3}, \mathrm{~d}^{3} p\right)=$ momentum space. This choice gives us a representation of $E_{3}^{*}$ which contains all momenta on an equal footing; to obtain an irreducible bundle representation we must take $\phi$ from $L_{2}\left(S_{2}\left(P^{2}\right), \mathrm{d} \omega\right)$ where $S_{2}\left(P^{2}\right)$ is the 2-sphere $P^{2}=\boldsymbol{p}^{2}$ and $d \omega$ an invariant measure on it; (iv) an irreducible representation of $E_{3}^{*}$ is characterized by the pair $\left(P^{2}, n\right)$. Hence in the representation (4.4) 
of $E_{3}^{*} \times T$ we must specify which representation of $T$ we are choosing for given $\left(P^{2}, n\right)$, i.e.

$$
E=E\left(P^{2}, n\right)
$$

in (4.4).

Finally, the explicit form of the bundle representation is:

$$
(b, \boldsymbol{a}, \boldsymbol{v}, R)(\boldsymbol{w}, \phi)=\left(\boldsymbol{v}+R \boldsymbol{w}, \exp i[\Lambda+n r(\omega, R)]\left(\phi \circ R^{-1}\right)(\boldsymbol{p})\right),
$$

where

$$
\Lambda=-\boldsymbol{a} \cdot \boldsymbol{p}+b\left\{E\left(P^{2}, n\right)+(\boldsymbol{v}+R \boldsymbol{w}) \cdot \boldsymbol{p}\right\} .
$$

The Landau excitations in superfluid helium correspond to $n=0$ and $E\left(P^{2}\right)$ the Landau spectrum in the rest frame.

These bundle representations have the following properties. (a) The background - i.e. the state in the absence of excitations - is invariant under $E_{3}^{*} \times T$. Its "state" is labelled by the single classical global observable $\boldsymbol{w}$. (b) The excitations have the boost properties characteristic of zero-mass in nonrelativistic physics. (c) The symmetry principle does not impose a dispersion law; the possibility of a dispersion law arises from the reducibility of the representation of $E_{3}^{*} \times T$. (d) In the presence of excitations, there exists a unique fibre which is Euclidean-invariant; it is the rest-fibre.

\subsection{Quasi-Particles in Translation-invariant Backgrounds}

We will now consider the situation in which the background remains $E_{3}^{*} \times T$ invariant, but the elementary excitations have mass. One may keep an ideal Fermi-liquid theory in mind as a model. The only change from the previous section consists in replacing $G_{3}^{*}$ by $\tilde{G}_{3}^{*}$ and $E_{3}^{*} \times T$ by $\Theta \times E_{3}^{*} \times T$, where $\Theta$ is the group which extends $G_{3}^{*}$. Since $\Theta$ is normal in $G_{3}^{*}$, the manifold $M=G / H$ remains unchanged. Again there exists a natural cross-section

$$
\eta(\boldsymbol{w})=(0,0,0, \boldsymbol{w}, 1)
$$

and (4.3) is replaced by

$$
k(\tilde{g}, \boldsymbol{w})=\left(\theta+\frac{1}{2} b(\boldsymbol{v}+R \boldsymbol{w})^{2}-(\boldsymbol{v}+R \boldsymbol{w}) \cdot \boldsymbol{a}, b, \boldsymbol{a}-b(\boldsymbol{v}+R \boldsymbol{w}), 0, R\right) .
$$

We choose the following representation of $\Theta \times E_{3}^{*} \times T$ on $L_{2}\left(\mathbb{R}^{3}, \mathrm{~d}^{3} p\right)$ :

$$
U(\theta, b, \boldsymbol{a}, \boldsymbol{v}, R) \phi(\boldsymbol{p})=\exp i m \theta \exp i[n r(\omega, R)+E b-\boldsymbol{p} \cdot \boldsymbol{a}] \phi \circ \boldsymbol{R}^{-1}(\boldsymbol{p})
$$

where all familiar symbols are as before, including $E=E\left(P^{2}, n\right)$ and the only new symbol $m$ is a real number which characterizes a representation of $\Theta$. By arguments similar to those which led to (4.5), we have here

$$
m=m\left(P^{2}, n\right) \text {. }
$$

We are interested only in the elements of the subgroup $G_{3}^{*}$ in the representations of $\tilde{G}_{3}^{*}$. For these elements, the explicit form of the bundle representation is

$$
(0, b, \boldsymbol{a}, \boldsymbol{v}, R)(\boldsymbol{w}, \phi)=\left(\boldsymbol{v}+R \boldsymbol{w}, \exp i[M+n r(\omega, R)]\left(\phi \circ R^{-1}\right)(\boldsymbol{p})\right)
$$

where

$$
M=-\boldsymbol{a} \cdot\left\{\boldsymbol{p}+m(\boldsymbol{v}+R \boldsymbol{w})+b\left\{E\left(P^{2}, n\right)=(\boldsymbol{v}+R \boldsymbol{w}) \cdot \boldsymbol{p}+\frac{m}{2}(\boldsymbol{v}+R \boldsymbol{w})^{2}\right\} .\right.
$$


One sees immediately that the energy and momentum now transform under boosts in the familiar manner of particles with mass $m$, so that

$$
E-\frac{p^{2}}{2 m}
$$

is independent of the fibre. However, it depends on $p^{2}$ and $n$ through $E$ and $m$, and therefore cannot be legislated away, as in ordinary nonrelativistic quantum mechanics.

It is easy to verify that Bargmann's superselection rule still holds, so that a dependence of $m$ on $P^{2}$ and $n$ may have drastic consequences for localizability.

Since $E, m$ and $\boldsymbol{p}$ are no longer related by the free-particle dispersion law, one has the possibility of identifying these excitations with quasi-particles and quasi-holes in systems with an Euclidean invariant ground state.

\subsection{Lattice Systems; Phonons}

We will now consider the bundle representations of $G_{3}$ in which the subgroup $H=L \times T$, where $L$ is discrete. For simplicity this subgroup will be assumed to have no rotation elements, i.e. it will be a group of lattice translations alone.

Let $\boldsymbol{u}_{1}, \boldsymbol{u}_{2}, \boldsymbol{u}_{3}$ be the three fundamental translations of $L$, and $u_{1}, u_{2}, u_{3}$ their respective magnitudes. The generic element of $L$ is $\boldsymbol{l}=n_{1} \boldsymbol{u}_{1}+n_{2} \boldsymbol{u}_{2}+n_{2} \boldsymbol{u}_{3}$, where $n_{1}, n_{2}, n_{3}$ are integers, $+v e,-v e$ or zero.

The homogeneous space $\Sigma=S_{3} / L$ is a three-dimensional torus. Let the coordinates of a point $\sigma \in \Sigma$ be $\sigma_{1}, \sigma_{2}, \sigma_{3}$, where

$$
-\frac{1}{2} u_{i}<\sigma_{i} \leqq \frac{1}{2} u_{i}, \quad i=1,2,3 .
$$

Then $\Sigma$ is mapped $(1,1)$ into the half-open unit cell $Q$ in $S_{3}$. Denote by $\sigma$ the translation in $Q$ which is the image of $\sigma \in \Sigma$. Then $\sigma$ has the components $\left(\sigma_{1}, \sigma_{2}, \sigma_{3}\right)$. Every translation $\boldsymbol{a}=(0, \boldsymbol{a}, 0,1) \in S_{3}$ has a unique decomposition

$$
\boldsymbol{a}=\boldsymbol{\sigma}(\boldsymbol{a})+\boldsymbol{l},
$$

where $\boldsymbol{\sigma}(\boldsymbol{a}) \in Q$ and $\boldsymbol{l} \in L$. The map $\pi_{\Sigma}: S_{3} \rightarrow \Sigma$ given by

$$
\pi_{\Sigma}(\boldsymbol{a})=\sigma(\boldsymbol{a})
$$

is continuous. Define a map $\eta_{\Sigma}: \Sigma \rightarrow S_{3}$ by

$$
\eta_{\Sigma}(\sigma)=\sigma
$$

This map is $(1,1)$, continuous except on the coordinate surfaces $\sigma_{i}=\frac{1}{2} u_{i}$, and satisfies

$$
\pi_{\Sigma} \eta_{\Sigma}(\sigma)=\sigma
$$

Since $S_{3}$ is not a product bundle over $\Sigma$, there exists no continuous map $\eta_{\Sigma}: \Sigma \rightarrow S_{3}$ satisfying (4.18). Note that if $\sigma(\boldsymbol{\sigma}) \in \Sigma$ then also $\sigma(-\boldsymbol{\sigma}) \in \Sigma$. Furthermore, $\boldsymbol{\sigma}(\boldsymbol{a})$ satisfies

$$
\sigma(-a)=-\sigma(a)
$$


Every $g \in G_{3}$ can be factorized uniquely into an element of $V_{3} \wedge R_{3}$ (the carat denoting semidirect product) and an element of $S_{3} \times T$ :

$$
(b, \boldsymbol{a}, \boldsymbol{v}, R)=(0,0, \boldsymbol{v}, R)\left(b, R^{-1}(\boldsymbol{a}-b \boldsymbol{v}), 0,1\right) .
$$

Hence

$$
M=G_{3} / L \times T=\left(V_{3} \wedge R_{3}\right) \cdot\left(S_{3} \times T / L \times T\right)=\left(V_{3} \wedge R_{3}\right) \cdot\left(S_{3} / L\right) .
$$

Thus a point $x \in M$ can be written explicitly as

$$
x=[w, \varrho ; s]
$$

where $w \in V_{3}, \varrho \in R_{3}$ and $s \in \Sigma$. The natural projection $\pi: G \rightarrow M$ is [cf. 4.20]

$$
\pi(b, \boldsymbol{a}, \boldsymbol{v}, R)=\left[\boldsymbol{v}, R ; \sigma\left(R^{-1}(\boldsymbol{a}-b \boldsymbol{v})\right)\right]
$$

where $\sigma(\boldsymbol{a})$ is defined by (4.15). The natural action of $G$ on $M$ is

$$
(b, \boldsymbol{a}, \boldsymbol{v}, R)[\boldsymbol{w}, \varrho ; s]=\left[\boldsymbol{v}+R \boldsymbol{w}, R \varrho ; \sigma\left\{(R \varrho)^{-1}(\boldsymbol{a}+R \varrho s+b \boldsymbol{v}-b R \boldsymbol{w})\right\}\right] .
$$

Finally, let $\eta: M \rightarrow G$ be defined by

$$
\eta[w, \varrho ; s]=(0, \varrho s, w, \varrho) .
$$

Then $\pi \circ \eta[w, \varrho ; s]=[w, \varrho ; \sigma(s)]$. But $s \in \Sigma$, and therefore $\sigma(s)=s$, and hence

$$
\pi \eta(x)=x, \forall x \in M \text {. }
$$

Let $g=(b, \boldsymbol{a}, \boldsymbol{v}, R), x$ be given by (4.21), and introduce the abbreviations

$$
\begin{aligned}
\Omega & =R Q \\
\boldsymbol{W} & =\boldsymbol{v}+R \boldsymbol{w} \\
\boldsymbol{A} & =\Omega^{-1}(\boldsymbol{a}+\Omega s-b \boldsymbol{W}) .
\end{aligned}
$$

Then

$$
k(g, x)=\eta(g x)^{-1} g \eta(x)=(b, A-\sigma(A), 0,1),
$$

which lies in $L \times T$ as $\boldsymbol{A}-\boldsymbol{\sigma}(\boldsymbol{A}) \in L$ for all $\boldsymbol{A}$.

The irreducible representations of $L \times T$ are characterized by points on $\hat{L} \times \hat{T}$, where the $\hat{L}$ is the Pontrjagin dual group of $L$ (and $\hat{T}$ that of $T$ ). As is usual, we shall denote a point on $\hat{L}$ by $\boldsymbol{k}$ and one on $\hat{T}$ by $E$. As $G$ is not a product bundle over $G / L \times T$, the canonical representations on $(G / L \times T) \times \mathscr{H}$ discussed in $\S 3.2$ will not be continuous if the representation of $L \times T$ on $\mathscr{H}$ is irreducible, i.e. onedimensional.

Continuous bundle representations can be constructed as follows. First observe that $G$ is not a product bundle over $G / L \times T$ only because $S_{3}$ is not a product bundle over $\Sigma$. However, the space $\Sigma$ is homeomorphic to $\hat{L}$, which also is topologically a 3-torus. It follows from the remarks in Chapter 3 that, by exploiting this homeomorphism, we can construct continuous bundle representations with fibre $\mathscr{H}=L_{2}(\hat{L}, \mathrm{~d} \mu)$, where $\mathrm{d} \mu$ is an $L$-invariant measure on $\hat{L}$. Hence let $\phi \in L_{2}(\hat{L}, d \mu)$ and choose the following representation of $L \times T$ :

$$
(b, \boldsymbol{l}, 0,1) \phi(\boldsymbol{k})=\exp i[E(\boldsymbol{k}) b-\boldsymbol{l} \cdot \boldsymbol{k}] \phi(\boldsymbol{k}),
$$

where $\boldsymbol{l} \in L, \boldsymbol{k}=\left(k_{1}, k_{2}, k_{3}\right) \in \hat{L}$ and $E(\boldsymbol{k})$ is an arbitrary real function of $\boldsymbol{k}$. 
The general formula for the bundle representation is cumbersome, and therefore we write down the following special cases from which the transformation properties of energy and momentum can be seen at a glance:

$$
\begin{aligned}
& (b, \boldsymbol{a}, 0,1)\{[0,1 ; 0], \phi\}=\{[0,1 ; \sigma(\boldsymbol{a})], \exp i[E(\boldsymbol{k}) b-\boldsymbol{k} \cdot(\boldsymbol{a}-\boldsymbol{\sigma}(\boldsymbol{a}))] \phi(\boldsymbol{k})\} . \\
& (b, \boldsymbol{a}, 0,1)\{[\boldsymbol{w}, 1 ; 0], \phi\} \\
& \quad=\{[\boldsymbol{w}, 1 ; \sigma(\boldsymbol{a}-b \boldsymbol{w})], \exp i[E(\boldsymbol{k}) b-\boldsymbol{k} \cdot(\boldsymbol{a}-b \boldsymbol{w}-\boldsymbol{\sigma}(\boldsymbol{a}-b \boldsymbol{w}))] \phi(\boldsymbol{k})\} .
\end{aligned}
$$

Setting $b=0$ in (4.29) and comparing with (4.28) we see that the momentum is unchanged by a boost. Setting $\boldsymbol{a}=0$ we find that the factor of $\boldsymbol{k}$ in the exponent becomes

$$
b \boldsymbol{w}+\boldsymbol{\sigma}(-b \boldsymbol{w}) .
$$

This is not generally linear in $b$. However, to obtain the transformation properties of the energy under boosts it is sufficient to consider infinitesimal time translations. As the above term vanishes for infinitesimal $b$, we conclude that the energy is invariant under boosts.

This conclusion is consistent with the usual "mechanical" interpretation of phonons as quantized lattice vibrations. However, in the mechanical interpretation the symmetry of the lattice ceases to be a rigorously valid concept at finite temperatures, which does not appear to be the case in the present formulation.

\subsection{Finite-Mass Excitations in a Lattice}

The considerations of the previous section can easily be extended to $\tilde{G}$. Taking $H$ to be $\Theta \times L \times T$, we see that the manifold $M$ is unchanged. The same is true for the action of the group on the manifold. The map $\tilde{\pi}: \tilde{G} \rightarrow M$ is now

$$
\tilde{\pi}(\theta, b, \boldsymbol{a}, \boldsymbol{v}, R)=\left[\boldsymbol{v}, R ; \sigma\left(R^{-1}(\boldsymbol{a}-b \boldsymbol{v})\right)\right]
$$

and $\tilde{\eta}: M \rightarrow \tilde{G}$ is defined by

$$
\tilde{\eta}(x)=\tilde{\eta}[\boldsymbol{w}, \varrho ; s]=(0,0, \varrho s, \boldsymbol{w}, \varrho) .
$$

Then $\tilde{\pi} \tilde{\eta}(x)=x$. We employ the notations of (4.25) to write down

$$
k(\tilde{g}, x)^{\prime}=\tilde{\eta}(\tilde{g} x)^{-1} \tilde{g} \tilde{\eta}(x)=(\tilde{\theta}, b, \boldsymbol{A}-\boldsymbol{\sigma}(\boldsymbol{A}), 0,1)
$$

where

$$
\tilde{\theta}=\left(\theta+\frac{1}{2} b \boldsymbol{v}^{2}-\boldsymbol{a} \cdot \boldsymbol{v}\right)-\boldsymbol{w} \cdot \Omega(\boldsymbol{A}-\boldsymbol{\sigma}(\boldsymbol{A}))+\boldsymbol{v} \cdot \Omega \boldsymbol{A} .
$$

Let us choose a one-dimensional representation of $\Theta \times L \times T$ :

$$
U(\theta, b, \boldsymbol{l}, 0,1)=\mathrm{e}^{i m \theta} \mathrm{e}^{i(b E-\boldsymbol{k} \cdot \boldsymbol{l})}
$$

where $E \in \hat{T}, \boldsymbol{k} \in \hat{L}$ and $\boldsymbol{l} \in L$. Here

$$
E=E(\boldsymbol{k})
$$

and

$$
m=m(\boldsymbol{k}) .
$$


The complete representation of $G$ on $M \times \mathscr{H}$ is given by the standard formula. As before, we shall write down only special cases of interest.

Take $\varrho=1$ and $s=0$ on $M$ and $\boldsymbol{v}=0$ and $R=1$ in $\tilde{g}$. Then

$$
(0, b, \boldsymbol{a}, 0,1)\{[0,1 ; 0], 1\}=\{[0,1 ; \sigma(\boldsymbol{a})], \exp i[E b-\boldsymbol{k} \cdot(\boldsymbol{a}-\boldsymbol{\sigma}(\boldsymbol{a}))]\} .
$$

$(0, b, \boldsymbol{a}, 0,1)\{[\boldsymbol{w}, 1 ; 0], 1\}=\{[\boldsymbol{w}, 1 ; \sigma(\boldsymbol{a})], \exp i[E b-(\boldsymbol{k}+m \boldsymbol{w}) \cdot(\boldsymbol{a}-b \boldsymbol{w}-\boldsymbol{\sigma}(\boldsymbol{a}-b \boldsymbol{w}))]\}$.

We see that the momentum $\boldsymbol{k}$ in the rest-frame transforms to

$$
\boldsymbol{k}^{\prime}=\boldsymbol{k}+m \boldsymbol{w}
$$

in the $w$-fibre. Considering, as before, only infinitesimal time translations, we find again that

$$
E^{\prime}=E
$$

i.e. the energy is also invariant under finite boosts. This is an effect of the lattice structure.

One verifies that Bargmann's superselection rule remains valid.

\section{§ 5. The Poincaré Group and Homogeneous Media}

If one restricts oneself to a fixed frame of reference there is no distinction between the Galilei and the Poincare group, when boosts are $\pi$-broken. A distinction only appears for physics in moving media, that is if we have to compute dragging coefficients.

In order to have some further motivation for this section we will touch upon the question of localizability. This is the following. The subgroup which leaves the medium at rest is the group of Euclidean transformations and time translations. Its irreducible representations are classified by energy, (momentum) ${ }^{2}$ and helicity. Thus the natural representations are obtained on the momentum space and act locally on this space. However for applications we usually need a representation where $x$-space functions appear and we would prefer to have the group act locally on this representation as well. Therefore we have to produce a representation "commuting" with Fourier transformations. That this can be done we know from experience. We shall exploit this empirical fact and shall not present a theory for this. Moreover we shall stick to the physically reasonable cases of spin $0, \frac{1}{2}$ and 1 , and shall deal here with the Poincaré group in order to avoid repetition.

\subsection{Spin Zero Systems}

The subgroup which leaves the background at rest is the Euclidean group times the time translations $E_{3} \times T$. The quotient space $P / E_{3} \times T$ is the space of the boosts, where $P$ denotes the Poincaré group. For obvious reasons we take the first column of the Lorentz matrix to characterize a pure Lorentz transformation. Then we have

$$
P / E_{3} \times T \cong H_{1}=\left\{b ; b^{2}=1, b_{0}>0\right\}
$$

where $b=\left\{b_{0}, \boldsymbol{b}\right\}$ and $b^{2}=b_{0}^{2}-\boldsymbol{b}^{2}$, the Minkowski square. On this manifold we have a natural action of the group $P$

$$
(a, \Lambda) b=\Lambda b,
$$


and a natural cross-section

$$
\eta(b)=(0, \Lambda(b))
$$

where $\Lambda(b)$ denotes the pure Lorentz transformation defined by $b$. From this we get according to (3.8)

$$
k((a, \Lambda), b)=\left(\Lambda^{-1}(\Lambda b) a, \Lambda^{-1}(\Lambda b) \Lambda \Lambda(b)\right) .
$$

The irreducible representations of $E_{3} \times T$ without angular momentum are characterized by the discrete eigenvalue $\varepsilon$ of $T$ and the continuous eigenvalues $\boldsymbol{k}$ of the translations in $E_{3}$ which lie on the sphere $\boldsymbol{k}^{2}=\varkappa^{2}$. When $\varkappa^{2} \neq 0$ our Hilbert space consists of the square integrable functions $\psi(\boldsymbol{k})$ on the sphere.

Since we are dealing with the relativistic case it is natural to take $\varepsilon$ and $\boldsymbol{k}$ together as a four-vector $p=b_{0} \varepsilon+\boldsymbol{k}$ where $b_{0}$ represents the zero boost. Then we have

$$
p^{2}=\varepsilon^{2}-\varkappa^{2}=m^{2} \text {. }
$$

Now the three-vector $\boldsymbol{k}$ and the four-vector $p$ are in one to one correspondence. We have replaced the sphere by the intersection of the hyperboloid $p^{2}=m^{2}$ with the hyperplane $(b, p)=\varepsilon$. The advantage of this procedure is obvious since we are now working with functions $\psi(p)$ and have a natural action of the group $P$ by

so that we get

$$
(a, \Lambda) \psi(p)=\exp i\left(a, \Lambda^{-1} p\right) \psi\left(\Lambda^{-1} p\right)
$$

$$
(a, \Lambda)(b, \psi(p))=\left(\Lambda b, \exp i\left(a, \Lambda^{-1} p\right) \psi\left(\Lambda^{-1} p\right)\right)
$$

In the frame characterized by $b$ the function $\psi(p)$ satisfies the equations

$$
(b, p) \psi(p)=\varepsilon \psi(p)
$$

and

$$
p^{2} \psi(p)=m^{2} \psi(p)
$$

This is a description which remains local after Fourier transformation. This description has taken such a simple form because we have not enforced the product structure on the bundle space: the measure defining the Hilbert space over $b$ is given by

$$
\delta\left(p^{2}-m^{2}\right) \delta((b p)-\varepsilon) \mathrm{d}^{4} p .
$$

\subsection{Spin 1 Excitations}

In the general situation we have to look for irreducible representations of the Euclidean group $E_{3}$ or its covering group $E_{3}^{*}$. Given a direction $\boldsymbol{k}$ on the sphere $\boldsymbol{k}^{2}=\varkappa^{2}$, its little group is the group of rotations around this direction. This is an abelian group which has the representations

$$
R(\varphi) \psi(\boldsymbol{k})=e^{i n \varphi} \psi(\boldsymbol{k})
$$

where

$$
n=0, \pm \frac{1}{2}, \pm 1, \ldots
$$

and $\varphi$ is the angle of rotation around the $\boldsymbol{k}$-axis. 
We are firstly interested in the cases $n= \pm 1$. In order to obtain an action of the rotation group which is independent of the choice of $\boldsymbol{k}$ one maps the function $\psi(\boldsymbol{k})$ onto a vector field $\boldsymbol{F}(\boldsymbol{k})$. To this end we choose two measurable real vector fields $\boldsymbol{e}_{1}(\boldsymbol{k}), \boldsymbol{e}_{2}(\boldsymbol{k})$ with $\left(\boldsymbol{k}, \boldsymbol{e}_{1}(\boldsymbol{k})\right)=\left(\boldsymbol{e}_{1}(\boldsymbol{k}), \boldsymbol{e}_{2}(\boldsymbol{k})\right)=0$ and $\boldsymbol{e}_{i}(\boldsymbol{k})^{2}=1$, which together with $(\boldsymbol{k} / x)$ form a right-handed coordinate system, and define

$$
\boldsymbol{F}_{ \pm}(\boldsymbol{k})=\frac{1}{\sqrt{2}}\left\{\boldsymbol{e}_{1}(\boldsymbol{k}) \mp i \boldsymbol{e}_{2}(\boldsymbol{k})\right\} \psi_{ \pm}(\boldsymbol{k}) .
$$

Then we get for a rotation around the $\boldsymbol{k}$-axis

$$
\left(\begin{array}{rr}
\cos \varphi & -\sin \varphi \\
\sin \varphi & \cos \varphi
\end{array}\right)\left(\begin{array}{l}
\left(\boldsymbol{F}_{ \pm}(\boldsymbol{k})\right)_{1} \\
\left(\boldsymbol{F}_{ \pm}(\boldsymbol{k})\right)_{2}
\end{array}\right)=e^{ \pm i \varphi}\left(\begin{array}{l}
\left(\boldsymbol{F}_{ \pm}(\boldsymbol{k})\right)_{1} \\
\left(\boldsymbol{F}_{ \pm}(\boldsymbol{k})\right)_{2}
\end{array}\right) .
$$

Knowing $\boldsymbol{F}_{ \pm}(\boldsymbol{k})$ we obtain $\psi(\boldsymbol{k})$ from the relations:

$$
\begin{aligned}
\psi_{ \pm}(\boldsymbol{k}) & =\frac{1}{\sqrt{2}}\left\{\left(\boldsymbol{e}_{1}(\boldsymbol{k}), \boldsymbol{F}_{ \pm}(\boldsymbol{k})\right) \mp i\left(\boldsymbol{e}_{2}(\boldsymbol{k}), \boldsymbol{F}_{ \pm}(\boldsymbol{k})\right)\right\} \\
0 & =\left(\boldsymbol{e}_{1}(\boldsymbol{k}), \boldsymbol{F}_{ \pm}(\boldsymbol{k})\right) \pm i\left(\boldsymbol{e}_{2}(\boldsymbol{k}), \boldsymbol{F}_{ \pm}(\boldsymbol{k})\right)
\end{aligned}
$$

The mapping $\psi_{ \pm}(\boldsymbol{k}) \rightarrow \boldsymbol{F}_{ \pm}(\boldsymbol{k})$ depends of the choice of the two vector fields $\boldsymbol{e}_{1}(\boldsymbol{k})$ and $\boldsymbol{e}_{2}(\boldsymbol{k})$, but this is of no importance since the scalar product

$$
\left(\psi^{(1)}, \psi^{(2)}\right)=\int \mathrm{d} \Omega_{k} \overline{\psi^{(1)}(\boldsymbol{k})} \psi^{(2)}(\boldsymbol{k})=\int \mathrm{d} \Omega_{k}\left(\boldsymbol{F}^{(1)}(\boldsymbol{k}), \boldsymbol{F}^{(2)}(\boldsymbol{k})\right)
$$

is independent of this choice.

What counts is the fact that we can characterize the spaces of vector fields $\boldsymbol{F}$ by the equations

$$
\left(\boldsymbol{k}, \boldsymbol{F}_{ \pm}(\boldsymbol{k})\right)=0 \quad \text { and } \quad\left[\boldsymbol{k}, \boldsymbol{F}_{ \pm}(\boldsymbol{k})\right]= \pm i \chi \boldsymbol{F}_{ \pm}(\boldsymbol{k})
$$

(where $[\cdot, \cdot]$ denotes the vector product) which go over into differential equations after Fourier transformations. For the representation of the group $E_{3} \times T$ we obtain

$$
(a, R) \boldsymbol{F}_{ \pm}(\boldsymbol{k})=\exp i\left(a_{0} \varepsilon-\left(\boldsymbol{a}, R^{-1} \boldsymbol{k}\right)\right)\left(R \boldsymbol{F}_{ \pm}\right)\left(R^{-1} \boldsymbol{k}\right)
$$

which also stays local after Fourier transformation.

In order to transform these equations into other frames, we have to pass to quantities which live in the four dimensional Minkowski space. This procedure is not unique, and we will choose such quantities for which the equations (4.14) remain simple.

We introduce two skew-symmetric tensors of second rank which have the following forms in the rest frame:

$$
F_{\mu \nu}=\left(\begin{array}{cccc}
0 & 0 & 0 & 0 \\
0 & 0 & F_{3} & -F_{2} \\
0 & -F_{3} & 0 & F_{1} \\
0 & F_{2} & -F_{1} & 0
\end{array}\right) \text { and } F_{\mu \nu}^{*}\left(\begin{array}{cccc}
0 & F_{1} & F_{2} & F_{3} \\
-F_{1} & 0 & 0 & 0 \\
-F_{2} & 0 & 0 & 0 \\
-F_{3} & 0 & 0 & 0
\end{array}\right)
$$

$\left(F^{*}\right.$ denotes the dual tensor of $\left.F\right)$. 
In terms of these quantities the equations of motion take the form

$$
b^{v} F_{v \mu}^{ \pm}=0 \text { and } \varepsilon p^{v} F_{v \mu}^{ \pm}= \pm i x p^{v}\left(F_{v \mu}^{ \pm}\right)^{*},
$$

and we obtain the bundle representation

$$
(a, \Lambda)\left(b, \boldsymbol{F}^{ \pm}(p)\right)=\left(\Lambda b, \exp i\left(a, \Lambda^{-1} p\right)\left(\Lambda \times \Lambda \boldsymbol{F}^{ \pm}\right)\left(\Lambda^{-1} p\right)\right) .
$$

With these Eqs. (5.17)-(18) we have obtained a scheme which stays local after the Fourier transformation.

\subsection{Hot Electrons}

In this section we want to investigate spin $\frac{1}{2}$ representations in the presence of a medium. Physically these representations have to be identified with electrons which feel only a bulk interaction with the background matter. They appear to correspond to the so-called "hot electrons".

Since we are dealing with spin $\frac{1}{2}$ systems, we have to map the function $\psi(\boldsymbol{k})$ in Eq. (5.9) onto spinor fields in order to get a representation which remains local after Fourier transformation.

Let us denote by $\boldsymbol{\sigma}$ the three Pauli matrices and $\psi_{ \pm}(\boldsymbol{k})$ the two spinors corresponding to the two eigenvalues $\pm \frac{1}{2}$. Then we conclude that in order to get the correct transformation, $\psi_{ \pm}(\boldsymbol{k})$ must be the eigenvectors of the matrix $\frac{1}{x}(\boldsymbol{k}, \boldsymbol{\sigma})$ with the eigenvalues \pm 1 . This gives us the equation of motion:

$$
(\boldsymbol{k}, \boldsymbol{\sigma}) \psi_{ \pm}(\boldsymbol{k})= \pm \chi \psi_{ \pm}(\boldsymbol{k})
$$

and the action of $E_{3}^{*} \times T$

$$
(a, A) \psi_{ \pm}(\boldsymbol{k})=\exp i\left(a_{0} \varepsilon-\left(\boldsymbol{a}, \boldsymbol{R}^{-1}(A) \boldsymbol{k}\right)\right)\left(A \psi_{ \pm}\right)\left(R^{-1}(A) \boldsymbol{k}\right) .
$$

In order to transform into other frames we will pass from the two-component spinors to four-component spinors by introducing

$$
\alpha_{0}=\left(\begin{array}{ll}
1 & 0 \\
0 & 1
\end{array}\right), \quad \alpha_{i}=\left(\begin{array}{rr}
\sigma_{i} & 0 \\
0 & -\sigma_{i}
\end{array}\right) \quad \text { and } \quad \gamma_{5}=\left(\begin{array}{rr}
1 & 0 \\
0 & -1
\end{array}\right) .
$$

Setting

$$
\Psi_{+}=\left(\begin{array}{c}
\psi_{+} \\
0
\end{array}\right) ; \quad \Psi_{-}=\left(\begin{array}{c}
0 \\
\psi_{-}
\end{array}\right)
$$

we obtain as equations of motion

$$
\gamma_{5} \Psi_{ \pm}(p)= \pm \Psi_{ \pm}(p)
$$

and

$$
\alpha_{v}\left(p^{v}+b^{v}(\varkappa-\varepsilon)\right) \Psi_{ \pm}(p)=0
$$

The last equation is the usual Dirac equation for massless particles, except for the replacement of $p$ by $p+b(x-\varepsilon)$. (For free neutrinos we have $x=\varepsilon$ ). The transformation law is now as usual:

$$
(a, A)(b, \Psi(p))=\left(\Lambda(A) b, \exp i\left(a, \Lambda^{-1}(A) p\right)(A \psi)(\Lambda(A) p)\right) .
$$


As we remarked in the beginning of this section, the representations with spin in the presence of matter will be interpreted in most cases as particles feeling only a bulk interaction with the medium. The most striking consequence of this theory is the fact that the spin lies either in the direction of the momentum or opposite to it. Furthermore these two spin directions are completely decoupled. This permits the possibility of having different dispersions for the two different spin orientations. This effect is known to occur in nature for photons, for which it is called the Faraday effect. But we should also expect the same effect to occur for hot electrons when passing through matter composed of magnetically asymmetric molecules.

Since, to our knowledge, this effect has not been observed, it might be of interest to give a detailed description.

We shall assume that a beam of coherent electrons passes through a layer of matter of thickness $d$, and that the direction of the beam is perpendicular to the surface. Since we assume the matter to be at rest, we can work with the Fourier transformed version of (5.19). For simplicity we shall assume that the direction of the beam is also the $z$-direction.

Since the boundary conditions (which we do not need to know explicitly) hold for all times, it follows that the energy is the same inside and outside of the matter. Only the absolute value $x$ of $\boldsymbol{k}$ can change. Since we are working with plane waves we get in the presence of matter the wave function

$$
\psi(z, t)=\left(\begin{array}{l}
\psi_{+} e^{-i x^{+} z} \\
\psi_{-} e^{-i \varkappa^{-} z}
\end{array}\right) \boldsymbol{e}^{i \varepsilon t}
$$

with $\left|\psi_{+}\right|^{2}+\left|\psi_{-}\right|^{2}=1$.

What one should observe is the spin component parallel to the surface. Computing the expectation values of $\frac{1}{2} \sigma_{x}$ and $\frac{1}{2} \sigma_{y}$ we get

$$
\begin{aligned}
& \left(\psi, \frac{1}{2} \sigma_{x} \psi\right)=\operatorname{Re}\left(\bar{\psi}_{+} \psi_{-} \boldsymbol{e}^{i\left(\varkappa^{+}-\varkappa^{-}\right) z}\right) \\
& \left(\psi, \frac{1}{2} \sigma_{y} \psi\right)=-\operatorname{Im}\left(\bar{\psi}_{+} \psi-\boldsymbol{e}^{i\left(\varkappa^{+}-x^{-}\right) z}\right)
\end{aligned}
$$

So we find that the spin component parallel to the surface is rotated by the amount

$$
d\left(x^{+}-x^{-}\right)
$$

proportional to the thickness of matter traversed.

\section{§ 6. Concluding Remarks}

In conclusion, we would like to remark upon the possible extensions and significance of this work.

1. It clarifies certain ideas concerning symmetries and broken symmetries. There is no need to discuss this further.

2. It provides a systematic approach to the study of phenomena in the presence of a medium, in all cases in which the medium can be characterized by a $\pi$-broken 
symmetry. The following points supplement the detailed discussion of the previous chapters:

(i) First, let only the boosts be $\pi$-broken. Then bundle representations provide a systematic theory of drag coefficients. In superfluidity, they provide the infrastructure for studying the Landau criterion. Among possible elementary excitations in a superfluid there exist those with nonzero energy and angular momentum, and zero linear momentum. These objects might correspond to quantum vortices.

(ii) Next, consider lattice systems, in which most translations are $\pi$-broken. The group of $\pi$-preserved symmetries being discrete, the product of two representations will decompose into a sum and not an integral. To us this suggests the possibility of formation of Cooper pairs. The theory of superfluidity of ${ }^{3} \mathrm{He}$ would, from this point of view, appear to be of exceptional interest.

(iii) Lastly, let some rotation elements be "missing" from the symmetry, due to the presence of an external electric or magnetic field. Then ray representations of translation groups become admissible. The resulting constraints on the motion of a particle can be automatically incorporated via bundle representations; the fibre becomes smaller than the Hilbert space of a free particle in just the right manner.

In addition to the above, we would like to mention the following more general questions.

3. In the quantum-mechanical measurement theory as developed by Hepp [8], the "observer" is identified with an infinite system. Our formalism provides a description of the boost operation on such systems.

4. Since many phase transitions are associated with a change of symmetry, and since the bundle formalism appears to be well-adapted to describe the latter, one should be able to study, for example, the solid-gas phase transition from the change of symmetry aspect.

5. Since the success of the theory of condensed matter is based largely on the picture of elementary excitations, and since the elementary excitations do appear to have some fundamental significance, an $a b$ initio study of condensed matter as a gas of weakly interacting excitations ought to be rewarding. The first requirement for such a programme is a precise definition of elementary excitations, which as we have seen is provided by the relativity principle. The interaction between excitations will, of course, have to be an input, just as the van der Waals interaction is in ordinary statistical mechanics. In the picture of condensed matter as a gas of elementary excitation, a phase transition is associated with a breakdown of the picture itself. This is clearly related to the phenomenon of instability of elementary excitations, and is beyond the scope of our present considerations.

The sum up, it appears that the action of relativity groups can yield useful information even when the state space is not a symplectic manifold or a Hilbert space.

Acknowledgements: This work was carried out during the year 1973-74. The second author (R.N.S.) would like to thank the Deutsche Forschungsgemeinschaft for financial support, and the members of the Institute for Theoretical Physics, University of Göttingen for their warm hospitality and stimulating company. He would like to acknowledge his indebtedness to J. Zak, F. R. Miller and J. Yngvason for many useful discussions. 


\section{Appendix: Fibre Bundles}

The topological notion of a fibre bundle [8] is a generalization of the notion of the product of two spaces: a fibre bundle is locally, but not always globally, a product.

In a product there are two natural projections from the total (or product) space onto the two component spaces. In a bundle, one of these is sacrificed, and the other, $\pi: B \rightarrow X, \pi$ continuous, is called the projection. $B$ is the total or bundle space and $X$ is the base space. The set $Y_{x} \equiv \pi^{-1}(x) \equiv\{b ; b \in B, \pi(b)=x\}, x \in X$ is called the fibre over $x$. The analogue of the second component in the product is recovered by making all the $Y_{x}$ homeomorphic to each other, and thus to an abstract fibre $Y$.

The local product structure is part of the axioms and is formulated as follows: There exists an open cover $\left\{V_{j}\right\}$ of $X$, where $j \in J$, an index set, such that for every $j \in J$ there exists a homeomorphism

$$
\phi_{j}: V_{j} \times Y \rightarrow \pi^{-1}\left(V_{j}\right)
$$

satisfying

$$
\pi \phi_{j}(x, y)=x, \quad x \in V_{j}, y \in Y,
$$

and the compatibility conditions described below on intersections of two $V^{\prime}$ s. A $\phi_{j}$ is called a local trivialization.

The local trivializations are glued together by means of a topological group $G$, called the group of the bundle. This is done as follows. Define a map $\phi_{j, x}: Y \rightarrow \pi^{-1}(x)$ by setting $\phi_{j, x}(y)=\phi_{j}(x, y)$. Then if $x \in V_{i} \cap V_{j}$ the map $\phi_{j, x}^{-1} \circ \phi_{i, x}: Y \rightarrow Y$ is a homeomorphism. This homeomorphism is identified with the action of a unique element $g \in G$ on $Y$; that is, $G$ must have an effective action on $Y$, or $Y$ must be a $G$-space. The resulting maps $g_{j i}: V_{i} \cap V_{j} \rightarrow G$ defined by

$$
g_{j i}(x)=\phi_{j, x}^{-1} \circ \phi_{i, x}
$$

are assumed to be continuous in $G$. This completes the definition of the coordinate bundle $\left\{B, \pi, X, Y, G, V_{i}, \phi_{i}\right\}$.

Two coordinate bundles with the same bundle space, projection, base space, fibre and group, but with coordinate neighbourhoods and local trivializations $\left(V_{i}, \phi_{i}\right)$ and $\left(V_{j}^{\prime}, \phi_{j}^{\prime}\right)$ are called strictly equivalent if, for $x \in V_{i} \cap V_{j}^{\prime}$, the map $\bar{g}_{j i}(x)$ $\phi_{j, x}^{\prime-1} \circ \phi_{i, x}$ of $Y$ into $Y$ coincides with the operation of an element of $G$, and the maps $\bar{g}_{j i}: V_{i} \cap V_{j}^{\prime} \rightarrow G$ so obtained are continuous. A fibre bundle is a strict equivalence class of coordinate bundles.

Recall that a system of coordinate transformations in $X$ with values in a topological group $G$ is an indexed covering $\left\{V_{i}\right\}$ of $X$ and a collection of continuous maps $g_{j i}: V_{i} \cap V_{j}$ such that (no summation over repeated indices!)

$$
g_{j i}(x) g_{i k}(x)=g_{j k}(x), \quad x \in V_{i} \bigcap V_{j} \bigcap V_{k} .
$$

Clearly (A.2) is satisfied by the $g_{j i}$ defined by (A.1), and therefore every bundle over $X$ with group $G$ determines such a system of coordinate transformations. There exists a converse, but before we state this result we must define a notion of equivalence.

Let $\{B, \pi, X, \ldots\}$ and $\left\{B^{\prime}, \pi^{\prime}, X^{\prime}, \ldots\right\}$ be two coordinate bundles with the same group and fibre. A map $h: B \rightarrow B^{\prime}$ is said to be a bundle map if $(a)$ it carries 
a fibre $Y_{x}$ homeomorphically onto a fibre $Y_{x^{\prime}} ;$ (b) the base map $\bar{h}: X \rightarrow X^{\prime}$ so induced satisfies $\pi^{\prime} h=\bar{h} \pi$; (c) the map $\bar{g}_{k j}(x)=\phi_{k, x^{\prime}}^{\prime-1} h_{x} \phi_{j, x}$ of $Y$ into $Y$ coincides with the operation of an element of $G$; and (d) the map $g_{k j}: V_{j} \cap \bar{h}^{-1}\left(V_{k}^{\prime}\right) \rightarrow G$ so obtained is continuous.

Two coordinate bundles having the same base space, fibre and group but different $B, B^{\prime}$ are said to be equivalent if there exists a bundle map $h: B \rightarrow B^{\prime}$ which induces the identity map of the common base space.

Now we can state the (constructive) existence theorem of Steenrod: If $G$ is a topological transformation group of $Y$, and $\left\{V_{i}\right\},\left\{g_{i j}\right\}$ is a system of coordinate transformations in $X$ with values in $G$, then there exists a bundle with base space $X$, fibre $Y$, group $G$, and the coordinate transformations $\left\{g_{i j}\right\}$. Any two such bundles are equivalent.

It should be clear by now that a fibre bundle is a topologically interesting structure precisely when it is not a global product. Therefore there exist a large number of concepts and results which are aimed at clarifying the conditions under which a bundle is a global product.

A bundle is called trivial if there exists a unique natural homeomorphism from $B$ to $X \times Y$. In this case there is only one coordinate neighbourhood $V=X$, and $G$ consists of the identity alone. A more complicated case occurs when $B$ is homeomorphic to $X \times Y$, but not in any unique natural manner. Then one needs a coordinate system to describe this homeomorphism. This at once introduces a group, the group of coordinates transformations which preserve this homeomorphism, which now becomes the group of the bundle. In this case the bundle is called not trivial but trivializable. We have used the term product bundle to cover both cases.

A continuous map $f: X \rightarrow B$ satisfying $\pi f(x)=x$ is called a cross-section. The notion of cross-section is a generalization of the notion of graph.

A bundle is called a principal bundle if the fibre is the same as the group (and the group acts on the fibre by left-translations). A principal bundle can be obtained from any bundle as follows. Keep the same base, coordinate neighbourhoods, coordinate transformations and group as the original bundle, replace the fibre by the group itself, and use the Steenrod construction to obtain a (unique) bundle. This bundle is called the associated principal bundle of the original.

A principal bundle is equivalent to the product if and only if it admits a crosssection. Two bundles having the same base space, fibre and group are equivalent if and only if their associated principal bundles are equivalent. Therefore a bundle is equivalent to the product if and only if its associated principal bundle admits a cross-section. (Note that a bundle may admit a cross-section even if its associated principal bundle does not!)

A real vector bundle is a bundle in which the fibre is an $n$-dimensional real vector space and the group is $G L(n, \mathbb{R})$. A complex vector bundle has a complex vector space as fibre and the group of unitaries upon it as group. A Hilbert bundle has (an infinite-dimensional) Hilbert space as fibre and the group of unitaries upon it as group. This group is not a limit $(n \rightarrow \infty) U(n)$.

The proofs of the following theorems depend on some of the deepest results in fibre bundle theory.

(1) A bundle is equivalent to the product if its base space is paracompact and contractible to a point. 
(2) A bundle is equivalent to the product if its base space is paracompact and its group is contractible to a point.

It has been proved by Kuiper [10] that the unitary group of Hilbert space is contractible to a point. Therefore every Hilbert bundle over a paracompact base is equivalent to the product.

Finally, we will state a general result on topological groups. Let $G$ be a topological group, $H$ a closed subgroup of $G, G / H$ the homogeneous space with the usual topology, and $\pi: G \rightarrow G / H$ the map defined by $\pi(g)=g H$. A local crosssection $f$ of $H$ in $G$ is a continuous $(1,1)$ map from an open set $V \subset G / H$ (containing the point $x_{0}=H$ ) into $G, f: V \rightarrow G$, such that $\pi f(x)=x \forall x \in V$.

It is a remarkable fact that if $G$ and $H$ are as above and $H$ has a local crosssection in $G$, then $G$ is a fibre bundle over $G / H$ with respect to the natural projection $\pi: G \rightarrow G / H$. The fibre of the bundle is $H$, and the group is $H$ acting on the fibre by left-translations. That is, $G$ is a principal bundle.

This result is known as the bundle structure theorem.

It has been proved by Chevalley [11] that if $G$ is a Lie group and $H$ a closed subgroup of $G$, then $H$ has a local cross-section in $G$. Hence the bundle structure theorem applies to every Lie group and every closed subgroup of it.

If $G$ is a Lie group and $H$ a closed subgroup of $G$, then $G / H$ is paracompact. Hence $G$ is a product bundle over $G / H$ if either $G / H$ is contractible to a point, or if $H$ is contractible to a point. The bundle $G$ over $G / H$ has a global crosssection if and only if it is equivalent to the product.

The maps $\eta: G / H \rightarrow G$ which one encounters in the theory of induced representations have the property $\pi \eta(x)=x$ of cross-sections. If $G$ is Lie and $H$ is closed, $H$ always has a local cross-section in $G$. Therefore one can always choose an $\eta$ which is piecewise-continuous. This result is sufficient to ensure continuity of the representations in the inducing construction.

\section{References}

1. Sen, R. N.: The Galilei group and Landau excitations. In: Statistical Mechanics and Field Theory. Sen, R. N., Weil, C., Eds. Jerusalem: Israel Universities Press 1972

2. Landau, L. D.: J. Phys. V, 71 (1941)

3. Haag, R., Kastler, D.: J. Math. Phys. 5, 848 (1964)

4. Borchers, H. J.: Commun. math. Phys. 4, 315 (1967)

5. Borchers, H.J.: Über $C^{*}$-Algebren mit lokalkompakten Symmetriegruppen. Nachrichten der Akad. Wiss. Göttingen, II. Math.-Phys. Klasse, Nr. 1 (1973)

6. Fell, J.M. G.: Can. J. Math. 14, 237 (1962)

7. Dixmier, J.: Les $C^{*}$-algèbres et leurs representations. Paris: Gauthier-Villars 1964

8. Hepp, K.: Helv. Phys. Acta 45, 237 (1972)

9. Steenrod, N.: The topology of fibre bundles, Princeton, N.J.: Princeton University Press, 1951 (with an appendix added in November 1956). This book has been our main source for material on this subject

10. Kuiper, N.H.: Topology 3, 19 (1965)

11. Chevalley, C.: Theory of Lie Groups, p. 135. Princeton, N.J.: Princeton University Press 1946 